\title{
Sperm chromatin and DNA integrity, methyltransferase mRNA levels, and global DNA methylation in oligoasthenoteratozoospermia
}

\author{
Tahereh Rahiminia', Ehsan Farashahi Yazd ${ }^{2}$, Farzaneh Fesahat ${ }^{3}$, Mohammad Reza Moein ${ }^{1}$, Ali Mohammad Mirjalili', \\ Ali Reza Talebi ${ }^{1}$ \\ ${ }^{1}$ Research and Clinical Center for Infertility, ${ }^{2}$ Stem Cell Biology Research Center, Research and Clinical Center for Infertility, and ${ }^{3}$ Department of \\ Advanced Medical Sciences and Technology, Faculty of Paramedicine, Shahid Sadoughi University of Medical Sciences, Yazd, Iran
}

Objective: To investigate sperm chromatin/DNA integrity, global DNA methylation, and DNMT mRNA transcription in men with oligoasthenoteratozoospermia (OAT) compared with normozoospermic men.

Methods: Semen samples from 32 OAT patients who comprised the case group and 32 normozoospermic men who comprised the control group were isolated and purified using a standard gradient isolation procedure according to World Health Organization criteria. DNMT1, DN$M T 3 A$, and DNMT3B transcripts were then compared between groups using real-time quantitative reverse-transcription polymerase chain reaction. Global DNA methylation in sperm was determined by an enzyme-linked immunosorbent assay. Protamine deficiency and the proportion of apoptotic spermatozoa were evaluated using chromomycin A3 (CMA3), aniline blue (AB), and toluidine blue (TB) staining, as well as the terminal deoxynucleotidyl transferase dUTP nick end labeling (TUNEL) assay. The $p$-values $<0.05$ were considered to indicate statistical significance.

Results: Significantly higher proportions of $\mathrm{AB}+, \mathrm{TB}+, \mathrm{CMA} 3+$, and TUNEL+ spermatozoa, as well as DNMT3A and DNMT3B transcription, were found in the OAT group. Positive correlations were detected between sperm parameters, DNA/chromatin damage, and DNMT3A and DNMT3B transcripts. Global DNA methylation was significantly higher in the OAT patients and had a significant correlation with abnormal results of all sperm chromatin integrity tests, but was not associated with DNMT1, DNMT3A, or DNMT3B expression.

Conclusion: Oligoasthenoteratozoospermic men showed abnormal sperm parameters, abnormal chromatin/DNA integrity, and a higher global DNA methylation rate, as well as overexpression of DNMT mRNA.

Keywords: Methylation; Methyltransferase; Sperm; Transcripts

\section{Introduction}

Oligoasthenoteratozoospermia (OAT) is the most common cause of male subfertility, and affects approximately $30 \%$ of all infertile men [1]. It is known that during spermatogenesis, several modifications of

Received: Oct 28, 2017 · Revised: Dec 18, 2017· Accepted: Dec 26, 2017 Corresponding author: Ali Reza Talebi

Research and Clinical Center for Infertility, Shahid Sadoughi University of Medical Sciences, Yazd 999-89195, Iran

Tel: +98-353-824-7085 Fax: +98-353-824-7087 E-mail: prof_Talebi@hotmail.com

This is an Open Access article distributed under the terms of the Creative Commons Attribution Non-Commercial License (http://creativecommons.org/licenses/by-nc/4.0/) which permits unrestricted non-commercial use, distribution, and reproduction in any medium, provided the original work is properly cited. chromatin occur, enabling the development of specific epigenetic profiles in spermatozoa. Recent evidence has suggested that some epigenetic modifications in sperm, such as DNA methylation, may play an important role in the development of male fertility [2,3]. Significant changes in the methylation level of sperm samples in $22 \%$ of infertile men were found to lead to abortion. This supports the idea that epigenetic disorders may explain the high incidence of abortion in humans [4].

DNA methylation is an epigenetic modification catalyzed by DNA methyltransferases (DNMTs) that can effectively promote gene silencing. DMNTs are classified into three types of enzymes in mammals (DNMT1, DNMT2, and DNMT3) [5]. It has been demonstrated 
that these enzymes are robustly expressed in the early embryonic stage [6]. DNMT1 is the most common enzyme, and plays a key role in the methylation of hemimethylated $\mathrm{CpG}$ dinucleotides in the mammalian genome; it is considered to be the key maintenance methyltransferase during cell division, whereas the function of DNMT3 is de novo methylation [7]. Some studies have demonstrated that methylation was significantly reduced in all CpGs in oligoasthenoteratozoospermic men, suggesting an association of abnormal DNA methylation-mediated genomic imprinting with OAT $[8,9]$. Furthermore, abnormal DNA methylation may be associated with the abnormal activation of DNMTs [10,11].

Higher levels of sperm nuclear organization occur shortly after fertilization, and are important for initiating and regulating the activity of the paternal gene in the early embryo [12]. Improper methylation is exhibited in more than $20 \%$ of sperm samples, which exhibit low concentrations, reduced sperm motility, and abnormal sperm morphology [4]. Sperm DNA fragmentation has been found to increase with increasing global methylation in infertile men [13]. Similarly, a correlation between the global methylation level and the status of chromatin injury detected by the aniline blue (AB) test was observed in samples from OAT patients [14], although the previous data showed a weak relationship of global methylation with sperm quality and DNA fragmentation [15].

Therefore, the literature contains insufficient evidence regarding the relationships of the transcription of DNMTs, such as DNMT1, DN$M T 3 A$, and DNMT3B, with sperm DNA global methylation and chromatin integrity. In the present study, we investigated various aspects of sperm chromatin/DNA integrity, global DNA methylation, and DNMT transcripts in men with OAT, and evaluated their relationships.

\section{Methods}

\section{Participants of the study}

In this prospective clinical study, semen samples were collected from 64 men referred to the andrology lab of the Yazd Research and Clinical Center for Infertility for infertility treatment. The participants were classified into two groups: 32 men with OAT in whom spermatogenetic disorders were detected in sperm analyses and who had a history of infertility, as a study group, and 32 normozoospermic men, who were the spouses in infertile couples with female etiology, as a control group. The inclusion criteria for patients was age $25-40$ years, sperm concentration 7-14 million/mL, $<40 \%$ total motility, and $<4 \%$ normal morphology. Heavy smokers (at least one pack of cigarettes per day during the past year), alcohol consumers (alcohol consumption during the last 3 months), and men with a history of varicocele were excluded from the study. This study was approved by the ethics committee of the Yazd Research and Clinical Center for In- fertility (No. 30710) and informed consent forms were signed by all participants. The study sampling (12 months) and the cellular/molecular studies (6 months) lasted from June 2015 to December 2016.

\section{Semen collection and determination of sperm parameters}

Semen samples from patients were collected by masturbation after 2-7 days of sexual abstinence. Samples were liquefied for at least 30 minutes at room temperature. Semen parameters were analyzed according to the strict World Health Organization (WHO) criteria (2010) [16]. Papanicolaou staining was performed to assess sperm morphology [17].

\section{Sperm chromatin and DNA integrity tests}

For the evaluation of sperm chromatin/DNA integrity, four tests were used: the terminal deoxynucleotidyl transferase dUTP nick end labeling (TUNEL) assay for DNA fragmentation, chromomycin A3 (CMA3) for sperm protamine deficiency, $A B$ staining for the detection of excessive histones in the process of chromatin condensation, and toluidine blue (TB) for sperm chromatin decondensation status and the exposure of phosphate groups [18].

\section{CMA3 staining}

Chromomycin A3 (Sigma, St. Louis, MO, USA) is a fluorochrome specific for guanosine and cytosine-rich sequences and is used to evaluate the degree of protamination of chromatin in sperm [19]. Sperm cells were fixed in Carnoy solution (methanol/glacial acetic acid, 3:1) at $48^{\circ} \mathrm{C}$ for 10 minutes. The slides were then stained with CMA3 solution $(0.25 \mathrm{mg} / \mathrm{mL}$ in Mcllvaine buffer; $7 \mathrm{~mL}$ of $0.1 \mathrm{M}$ citric acid+32.9

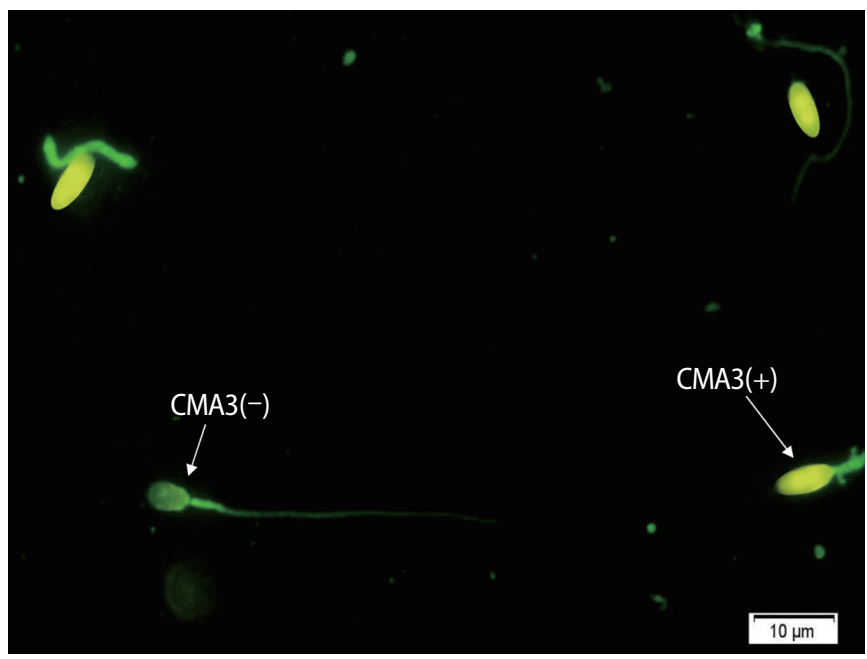

Figure 1. Chromomycin $\mathrm{A} 3$ (CMA3) staining for the evaluation of sperm protamine deficiency. Bright yellow sperm (CMA3+) shows protamine deficiency, while yellowish sperm (CMA3-) shows normal protamine content (fluorescent microscopy, $\times 100$ eyepiece magnification). 
$\mathrm{mL}$ of $0.2 \mathrm{M} \mathrm{Na}_{2} \mathrm{HPO}_{4} \cdot 7 \mathrm{H}_{2} \mathrm{O}$, pH 7.0 containing $10 \mathrm{mM} \mathrm{MgCl}$ ) for 20 minutes in a dark room. At least 200 spermatozoa were counted under florescent microscopy (BX51; Olympus, Tokyo, Japan) with a 460$\mathrm{nm}$ filter and $\times 100$ eyepiece magnification. The percentages of spermatozoa with bright yellow heads (CMA3+) and without brightness (CMA3-) were determined and reported (Figure 1) [20,21].

\section{TUNEL assay}

The percentage of apoptotic spermatozoa with DNA fragmentation

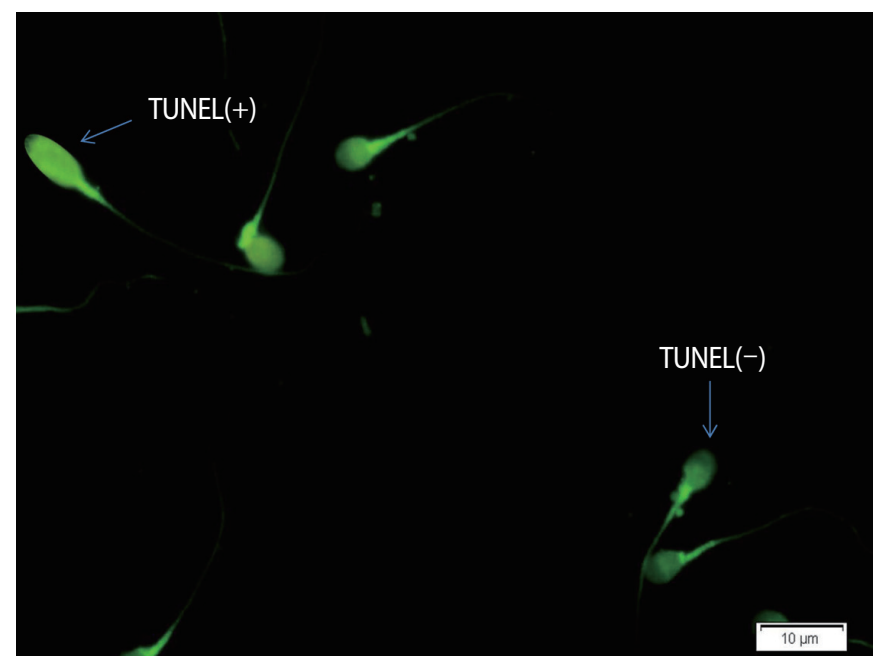

Figure 2. TUNEL assay for the detection of sperm DNA fragmentation. Under fluorescent microscopy, normal DNA is seen as light green (TUNEL-) and damaged DNA is seen as bright green (TUNEL+) ( $\times 100$ eyepiece magnification). TUNEL, terminal deoxynucleotidyl transferase dUTP nick end labeling.

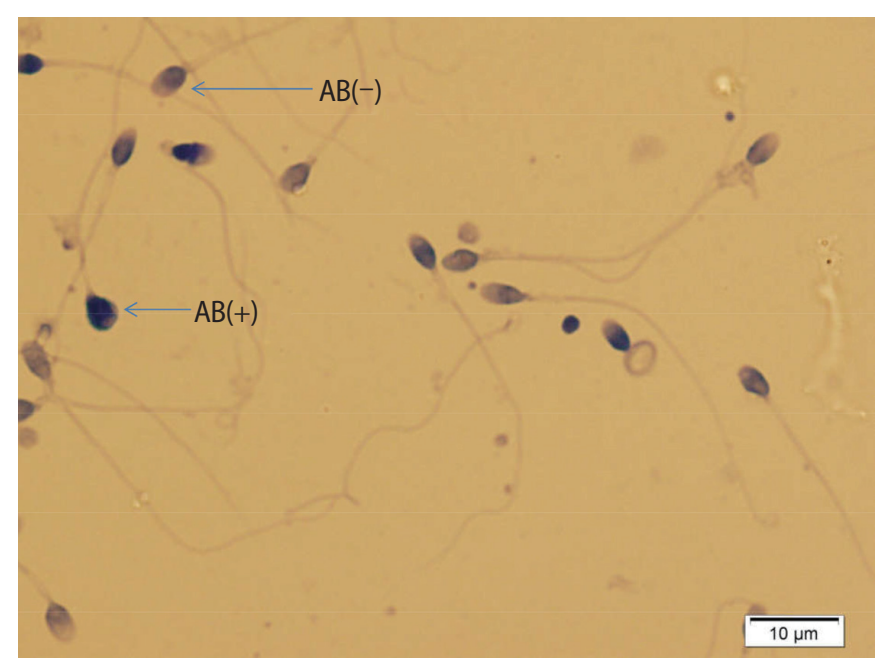

Figure 3. Aniline blue $(A B)$ staining for the detection of excessive histones in sperm chromatin. Pale blue stained spermatozoa $(A B-)$ are normal, and dark blue stained spermatozoa $(A B+)$ are considered to be abnormal. in each sample was determined by the TUNEL assay using an in situ cell death detection kit (Roche Diagnostics, Mannheim, Germany) using fluorescent microscopy, in which normal DNA was shown as light green and damaged DNA was seen as bright green (Figure 2) [22].

\section{AB staining}

$A B$ staining was performed as in our previous study. The air-dried smears were fixed in 3\% buffered glutaraldehyde in $0.2 \mathrm{M}$ phosphate buffer ( $\mathrm{pH}$ 7.2) for 30 minutes at room temperature. Each smear was stained with $5 \%$ aqueous $A B$ stain in $4 \%$ acetic acid ( $\mathrm{pH} 3.5)$ for 5 minutes. Unstained or pale blue stained spermatozoa ( $A B-$ ) were normal and dark blue stained spermatozoa $(A B+)$ were considered to be abnormal (Figure 3) [17,21].

\section{TB staining}

TB staining was done as described in our previous work. The dried smears were fixed by $96 \%$ ethanol-acetone $(1: 1)$ at $4^{\circ} \mathrm{C}$ for $30 \mathrm{~min}$ utes. The smears were hydrolyzed with $0.1 \mathrm{~N} \mathrm{HCl}$ at $4^{\circ} \mathrm{C}$ for 5 minutes and washed three times in distilled water. Then, staining was done with $0.05 \%$ TB (Sigma) for 10 minutes [21,23]. Pale blue sperm cells were considered to be normal, and dark blue or violet/purple spermatozoa were categorized as abnormal cells. For both tests, at least 200 spermatozoa were checked in each slide and the percentage of normal and abnormal spermatozoa was reported (Figure 4) [23,24].

\section{Sperm preparation by discontinuous density gradients}

We used the standard gradient isolation procedure described by the $\mathrm{WHO}[25]$ to isolate and purify the sperm samples of each group.

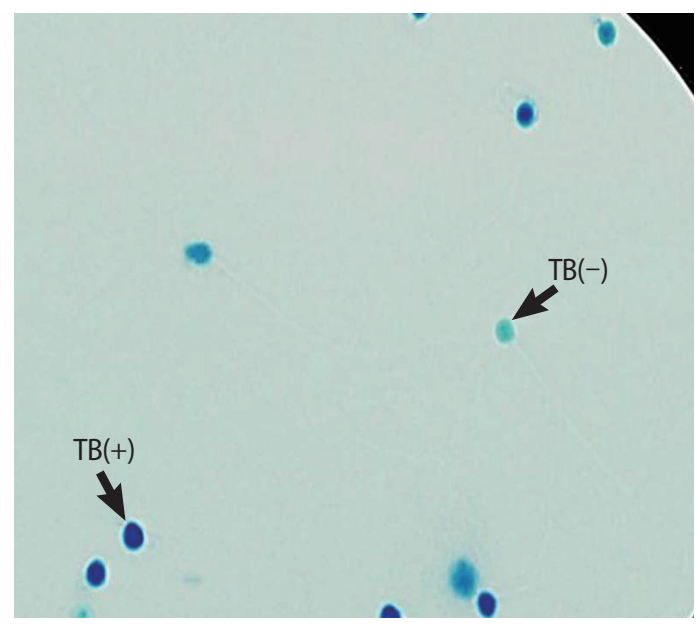

Figure 4. Toluidine blue (TB) staining to evaluate sperm chromatin decondensation status and the exposure of phosphate groups. Pale blue spermatozoa are considered to be normal (TB-), and dark blue or violet/purple spermatozoa are categorized as abnormal cells (TB+) (fluorescent microscopy, $\times 100$ eyepiece magnification). 
Briefly, a density gradient medium was prepared in a 15-mL test tube by layering $1 \mathrm{~mL}$ of $40 \%(\mathrm{v} / \mathrm{v}$ ) density-gradient medium over $1 \mathrm{~mL}$ of $80 \%$ (v/v) density gradient medium. About $1 \mathrm{~mL}$ of each well-mixed semen sample was placed on top of the density gradient media and then centrifuged at 0.4 relative centrifugal force (RCF) for 15 minutes. The supernatant was removed from the sperm pellet and mixed by gentle pipetting in $5 \mathrm{~mL}$ of Ham's F10 medium (Irvine Scientific, Santa Ana, CA, USA). The separated sperm pellet was centrifuged twice at $0.4 \mathrm{RCF}$ for 5 minutes. After resuspending the sperm pellet in 0.5 $\mathrm{mL}$ of phosphate-buffered saline, it was stored at $-80^{\circ} \mathrm{C}$ for further study.

\section{Global DNA methylation analysis}

For the global DNA methylation analysis, we used the 5-mC DNA ELISA Kit No. D5326 (Zymo Research Corp., Irvine, CA, USA). Briefly, $100 \mathrm{ng}$ of isolated denatured sperm DNA from each sample was dissolved in 5-methyl cytosine (5-mC) coating buffer and incubated at $37^{\circ} \mathrm{C}$ for 1 hour. Then, the sample was incubated at $37^{\circ} \mathrm{C}$ for $30 \mathrm{~min}-$ utes, followed by washing three times with $200 \mu \mathrm{L}$ of 5-mC enzymelinked immunosorbent assay (ELISA) buffer. Anti-5-methylcytosine and the secondary antibody were added to the 5-mC ELISA buffer and incubated at $37^{\circ} \mathrm{C}$ for 1 hour. Finally, horseradish peroxidase developer was added to each well after washing, and the wells were stored at room temperature for 20 minutes. For color detection, optical absorbance was measured at $450 \mathrm{~nm}$ using an ELISA plate reader (Star Fax-2100; Awareness Technology, Palm City, FL, USA). The global methylation assay for all samples was performed in duplicate, and the average value was shown with a generated standard curve using negative and positive internal controls on the same plate.

\section{Human sperm RNA extraction}

Total RNA was isolated from the collected sperm samples of each group using the RNeasy Plus Universal Mini Kit (Qiagen, Germantown, MD, USA) according to the manufacturer's protocol. The RNA concentration was measured using a NanoDrop spectrophotometer (2000c;
Thermo Scientific, Waltham, MA, USA). After RNA normalization of each sample, the complementary DNA (cDNA) was synthesized.

\section{Quantitative real-time reverse-transcription polymerase chain reaction}

Approximately $100 \mathrm{ng}$ of RNA was reverse-transcribed into CDNA using the RevertAid First Strand cDNA Synthesis kit (Thermo Scientific). Then, the quantitative polymerase chain reaction (PCR) was performed using the Fast SYBR Green master mix (Applied Biosystems, Foster City, CA, USA), in which 100 ng of CDNA per sample was used to amplify the target genes with the primers of the human genes DNMT1, DNMT3A, DNMT3B, and B2M (Table 1). In the thermal cycler (step 1, Applied Biosystems), the thermal cycling program included an initial incubation step at $95^{\circ} \mathrm{C}$ for 20 seconds, followed by 40 cycles of $95^{\circ} \mathrm{C}$ for 3 seconds and $58^{\circ} \mathrm{C}$ for 30 seconds. A final $58^{\circ} \mathrm{C}$ to $95^{\circ} \mathrm{C}$ step was used to create a melt curve. Two replicates for each reaction were performed and the threshold cycle $(\mathrm{Ct})$ values were averaged. The $2^{-\Delta c t}$ was calculated to represent the levels of gene expression after normalization to the levels of $B 2 M$, where $\Delta C t$ is defined as ( $C t$ genes-Ct B2M).

\section{Statistical analysis}

The nonparametric Mann-Whitney U-test was used to analyze differences in sperm parameters, mRNA expression, and global DNA methylation between the two study groups. The Spearman test was used to calculate correlations. All analyses were performed in PASW ver. 18.0 (SPSS Inc., Chicago, IL, USA). The $p$-values $<0.05$ were considered to indicate statistical significance.

\section{Results}

In this study, a total of 64 semen samples were studied in the two groups. There were no significant differences in age or body mass index between the groups. However, the percentages of sperm with normal morphology and progressive motility, as well as the sperm

Table 1. Primer sequences, sequence amplified, and product size of the genes included in this study

\begin{tabular}{|c|c|c|c|}
\hline Gene & Primer sequence $\left(5^{\prime}-3^{\prime}\right)$ & Sequence amplified & Product size (bp) \\
\hline \multirow[t]{2}{*}{ DNMT1 } & F-TGG ACG ACC CTG ACCTCA AAT & NM_001318731.1 & 168 \\
\hline & R-GCTTACAGTACA CACTGA AGC A & & \\
\hline \multirow[t]{2}{*}{ DNMT3A } & F-TATTGA GCG CAC AAG AGAG & NM_001320893.1 & 111 \\
\hline & R-GGGTGTTCC AGG GTA ACATTG AG & & \\
\hline \multirow[t]{2}{*}{ DNMT3B } & F-GGC AAGTTC TCC GAG GTC TCTG & NM_001207056.1 & 113 \\
\hline & R-TGG TAC ATG GCTTTTCGA TAGGA & & \\
\hline \multirow[t]{2}{*}{$B 2 M$} & F-AGATGAGTATGCCTGCCGTG & NM_004048.2 & 106 \\
\hline & R-TGCGGCATCTTCAAACCTC & & \\
\hline
\end{tabular}

$F$, forward; $R$, reverse. 
Table 2. Comparison of patients' characteristics, semen analysis results, chromatin/DNA status, and DNMT expression between the two groups

\begin{tabular}{|c|c|c|c|}
\hline Variable & Normozoospermia $(n=32)$ & Oligoasthenoteratozoospermia $(n=32)$ & $p$-value \\
\hline Age $(y r)^{a)}$ & $28 \pm 2$ & $27 \pm 2$ & 0.610 \\
\hline Body mass index $\left(\mathrm{kg} / \mathrm{m}^{2}\right)^{\mathrm{a})}$ & $23.4 \pm 1.5$ & $23 \pm 1.7$ & 0.701 \\
\hline Sperm count $\left(\times 10^{6} / \mathrm{mL}\right)^{\mathrm{b})}$ & $137(115-143)$ & $11.5(10-12.5)$ & $<0.001$ \\
\hline Progressive motility (\%) & $47(43-52)$ & $12.5(10.9-22)$ & $<0.001$ \\
\hline Normal morphology $(\%)^{b)}$ & $15(12.4-18)$ & $1(0.6-1.4)$ & $<0.001$ \\
\hline$A B(+)^{b)}$ & $25(24.5-29.5)$ & $43(41-46)$ & $<0.001$ \\
\hline $\mathrm{TB}(+)^{\mathrm{b})}$ & $40(35-39.9)$ & $63(58-66)$ & $<0.001$ \\
\hline TUNEL $(+)^{\mathrm{b})}$ & $10(9.2-10.2)$ & $18(17.4-19.7)$ & $<0.001$ \\
\hline $\operatorname{CMA} 3(+)^{\mathrm{b})}$ & $18(16.3-18.9)$ & $36(35-39)$ & $<0.001$ \\
\hline Global DNA methylation (\%) ${ }^{b}$ & $1.9(1.6-2.5)$ & $3.2(2.8-3.5)$ & $<0.001$ \\
\hline DNMT1 mRNA & $0.005(0.131-0.490)$ & $0.05(0.4-0.09)$ & 0.554 \\
\hline DNMT3A mRNA & $0.004(0.005-0.028)$ & $0.06(0.04-0.12)$ & 0.009 \\
\hline DNMT3B mRNA & $0.016(0.01-0.059)$ & $0.128(0.095-0.17)$ & 0.021 \\
\hline
\end{tabular}

Values are presented as mean \pm standard deviation or median (range).

$\mathrm{AB}$, aniline blue; TB, toluidine blue; TUNEL, terminal deoxynucleotidyl transferase dUTP nickend labeling; CMA3, chromomycin A3.

a)The $t$-test was used. The data distribution was normal; ${ }^{b}$ The Mann-Whitney $U$-test was used. The data distribution was not normal.

Table 3. Correlations between semen parameters and chromatin/DNA integrity, DNA methylation, and DNMT expression

\begin{tabular}{lcccccccc}
\hline Variable & $\mathrm{AB}(+)$ & $\mathrm{TB}(+)$ & TUNEL(+) & CMA3(+) & $\begin{array}{c}\text { Global DNA } \\
\text { methylation }\end{array}$ & $\begin{array}{c}\text { DNMT1 } \\
\text { mRNA }\end{array}$ & $\begin{array}{c}\text { DNMT3A } \\
\text { mRNA }\end{array}$ & $\begin{array}{c}\text { DNMT3B } \\
\text { mRNA }\end{array}$ \\
\hline Concentration & -0.67 & -0.67 & -0.71 & -0.767 & -0.41 & -0.08 & -0.34 & -0.34 \\
Progressive motility & $(<0.001)$ & $(<0.001)$ & $(<0.001)$ & $(<0.001)$ & $(<0.001)$ & $(0.5)$ & $(0.008)$ & $(0.008)$ \\
& -0.65 & -0.55 & -0.63 & -0.665 & -0.44 & -0.12 & -0.33 & -0.25 \\
Normal morphology & $(<0.001)$ & $(<0.001)$ & $(<0.001)$ & $(<0.001)$ & $(<0.001)$ & $(0.37)$ & $(0.01)$ & $(0.04)$ \\
& -0.67 & -0.74 & -0.71 & -0.730 & -0.34 & 0.009 & -0.26 & -0.19 \\
& $(<0.001)$ & $(<0.001)$ & $(<0.001)$ & $(<0.001)$ & $(0.008)$ & $(0.9)$ & $(0.04)$ & $(0.13)$ \\
\hline
\end{tabular}

The Spearman correlation test was used. In each cell, the top value is $r$ and the bottom is the $p$-value. The $p$-values $<0.05$ were considered to indicate statistical significance.

$A B$, aniline blue; TB, toluidine blue; TUNEL, terminal deoxynucleotidyl transferase dUTP nick end labeling; CMA3, chromomycin A3.

concentration, were statistically significantly different between the case and control groups. Significantly lower sperm concentration, progressive motility, and normal morphology were found in OAT patients than in the normozoospermic subjects. In addition, the proportions of spermatozoa with $\mathrm{AB}+, \mathrm{TB}+, \mathrm{CMA} 3+$ and TUNEL+ results were significantly higher in the OAT group than in the normozoospermia group, with meaningful implications for sperm DNA/chromatin integrity $(p<0.001)$ (Table 2$)$.

Regarding the methyltransferase mRNA levels, we observed significantly higher DNMT3A and DNMT3B transcriptional activity, as well as a nonsignificantly higher level of DNMT1, in OAT patients in comparison to normozoospermic subjects ( $p=0.009, p=0.021$, and $p=$ 0.554 , respectively) (Table 2). DNMT1 transcripts were not correlated with either sperm DNA/chromatin damage or sperm parameters. In contrast, positive correlations were found between sperm parameters, DNA and chromatin damage, and the quantity of DNMT3A and DNMT3B transcripts (Table 3).
Table 4. Correlations between chromatin/DNA integrity, global DNA methylation, and DNMT expression

\begin{tabular}{lcccc}
\hline Variable & $\begin{array}{c}\text { Global DNA } \\
\text { methylation }\end{array}$ & $\begin{array}{c}\text { DNMT1 } \\
\text { mRNA }\end{array}$ & $\begin{array}{c}\text { DNMT3A } \\
\text { mRNA }\end{array}$ & $\begin{array}{c}\text { DNMT3B } \\
\text { mRNA }\end{array}$ \\
\hline $\mathrm{AB}(+)$ & 0.41 & -0.05 & 0.39 & 0.38 \\
& $(0.001)$ & $(0.7)$ & $(0.002)$ & $(0.003)$ \\
$\mathrm{TB}(+)$ & 0.37 & -0.07 & 0.26 & 0.25 \\
& $(0.003)$ & $(0.59)$ & $(0.04)$ & $(0.04)$ \\
$\mathrm{TUNEL}(+)$ & 0.64 & 0.029 & 0.47 & 0.39 \\
& $(<0.001)$ & $(0.8)$ & $(0.002)$ & $(0.002)$ \\
CMA3(+) & 0.474 & 0.057 & 0.445 & 0.43 \\
& $(<0.001)$ & $(0.67)$ & $(0.001)$ & $(0.001)$ \\
Global DNA methylation & - & 0.17 & 0.22 & 0.16 \\
& & $(0.18)$ & $(0.08)$ & $(0.2)$ \\
\hline
\end{tabular}

The Spearman correlation test was used. In each cell, the top value is $r$ and the bottom is the $p$-value. The $p$-values $<0.05$ were considered to indicate statistical significance.

$A B$, aniline blue; $T B$, toluidine blue; TUNEL, terminal deoxynucleotidyl transferase dUTP nick end labeling; CMA3, chromomycin A3. 
Global DNA methylation in spermatozoa was significantly more extensive in the OAT patients than in the control group $(p<0.001)$. Global methylation showed a significant negative correlation with concentration, progressive motility, and normal morphology (Table 3). Our experiments demonstrated that global methylation had a significant correlation with abnormal results of all the sperm chromatin integrity tests, including TUNEL, AB, TB and CMA3. However, global methylation was not significantly associated with DNMT1, DNMT3A, or DNMT3B expression (Table 4).

\section{Discussion}

\section{Sperm DNA and chromatin integrity}

Our study demonstrated that men with OAT had a large proportion of sperm cells with excessive histones and protamine deficiency, as well as significantly elevated sperm DNA fragmentation, which is considered to be the main indicator of sperm apoptosis. The findings reflect a significant decrease in the proportion of spermatozoa with normal chromatin condensation in men with OAT, as has been previously reported $[26,27]$. To explain these results, it should be noted that the abnormal replacement of histones by protamine can increase sperm DNA fragmentation, and there is a clear relation between sperm chromatin quality and DNA damage [28].

\section{DNMT transcripts}

We found higher levels of DNMT3A and DNMT3B transcripts in the spermatozoa of the OAT subjects than in the control group. A study conducted by Marques et al. [29] on the expression of DNMTs in human spermatogenesis cells using quantitative reverse-transcription PCR confirmed the hypothesis of maintenance of DNA methylation at all stages of spermatogenesis, the results of which are manifested in the maintenance of paternal imprinting in the process of spermatogenesis [30].

\section{DNMT transcripts and sperm quality}

DNMT1 mRNA levels were not correlated with any of the sperm parameters. Decreased sperm concentration and progressive motility were significantly associated with increased DNMT3A and DNMT3B transcripts. Among the sperm parameters, only morphology was related to DNMT3A. Although no study has investigated the results of methyltransferases in relation to sperm analysis, the presence of a polymorphism in DNMT1 may be associated with oligospermia and sensitivity to lower sperm concentration [31].

In another report, the level of DNMT3A transcripts was identified as a marker of nonprogressive motile sperm. A significant increase in the transcription of DNMT3A, which is responsible for de novo DNA methylation, indicated the failure of sperm to obtain a suitable meth- ylation pattern for progressive motility [32].

\section{DNMT transcripts and chromatin and DNA integrity}

DNMT1 mRNA was not correlated with the results of sperm chromatin/DNA integrity tests, including TUNEL, AB, TB, and CMA3. However, abnormal results of these tests were correlated with increases in DNMT3A and DNMT3B mRNA. Increased DNMT3A and DNMT3B transcription was associated with more extensive sperm DNA fragmentation, protamine deficiency, excess histones, and exposure of the phosphate groups.

Although we did not find much information explaining these correlations and their mechanisms, it is possible to view these findings in light of the general relationships between chromatin integrity and the factors involved in epigenetic regulation. Some authors have shown that global genomic hypomethylation may be the primary response to oxidative stress, as mediated through increased DNMT activity [14]. Incomplete sperm nuclear compaction following the increased expression of histone deacetylase 1 and DNMT3A may affect the maturation of the sperm and be reflected in a reduction of chromatin integrity and sperm motility [32,33].

\section{Global DNA methylation}

Our results showed that DNA methylation levels were significantly higher in the OAT patients than in the control group. Yu et al. [33] showed that DNA methylation and histone retention levels were lower in selected sperm than in unprocessed sperm, which is similar to the findings of our study in terms of the increase in global DNA methylation and histone retention in men with OAT. Similarly, Barzideh et al. [34] showed that the quantity of 5-mC as a percentage of total cytosine was significantly lower in high-quality sperm, which was also confirmed by immunocytochemistry results.

\section{Global DNA methylation and sperm quality}

Higher global methylation levels in the OAT patients were associated with lower levels of concentration, progressive motility, and normal morphology. In contrast, Olszewska et al. [14] examined global methylation levels, estimating 5-mC levels in sperm with chromosomal abnormalities and in normal sperm, and showed that the DNA methylation levels were similar in both groups and that there were no correlations between sperm methylation, concentration, morphology, and progressive motility.

\section{Global DNA methylation and sperm DNA and chromatin integrity}

The increase in the patients' global methylation levels led to an increase in sperm chromatin/DNA dysfunction. Aoki and colleagues $[13,35]$ evaluated the relationship between the increased fragmenta- 
tion of DNA in infertile men and the increased global methylation of sperm DNA, and found a significant association with reduced semen quality and sperm chromatin/DNA integrity. Similarly, a correlational analysis between global methylation and the protamine levels of chromatin showed a positive association in OAT group [14], while a previous report showed a weak relationship of global methylation patterns with sperm quality and DNA fragmentation [15]. However, a tendency to undergo apoptosis is related to the state of global methylation of sperm DNA. Additionally, extensive denaturation of chromatin occurs in low-quality sperm. Such behavior probably enables the opening of the chromatin structure, allowing the anti-5-mC antibody to access methylation sites [29].

\section{Global DNA methylation and DNMT transcripts}

In our study, global methylation was not significantly associated with DNMT1, DNMT3A, or DNMT3B expression. In other tissues such as the placenta, methylation of the DNMT1 promoter reduces the transcriptional activity of this gene in trophoblast cells and results in hypomethylation of this tissue [36]. Despite more frequent alterations in DNA methylation in men with OAT [37], the probable effects of these defects on assisted reproductive technology outcomes are still unclear. Zama and Uzumcu [38] showed that OAT patients with partial hypomethylation of $\mathrm{H} 19$ demonstrated a lower fertilization rate following assisted reproductive technology.

In conclusion, we found that infertile patients with OAT showed abnormal sperm parameters, abnormal chromatin/DNA integrity, and a higher global DNA methylation rate, as well as overexpression of the mRNA of methyltransferase enzymes.

\section{Conflict of interest}

No potential conflict of interest relevant to this article was reported.

\section{Acknowledgments}

We would like to thank Mr. Mehrdad Soleimani, the head of the laboratories, as well as the staff of Assisted Reproductive Technologies, Andrology, Recurrent Abortion Study Research Center, and Hormone Laboratories in Research and Clinical Center for Infertility, Shahid Sadoughi University of Medical Sciences, Yazd, Iran.

\section{References}

1. Cavallini G. Male idiopathic oligoasthenoteratozoospermia. Asian J Androl 2006;8:143-57.

2. Komiya A, Kato T, Kawauchi Y, Watanabe A, Fuse H. Clinical factors associated with sperm DNA fragmentation in male patients with infertility. ScientificWorldJournal 2014;2014:868303.

3. Cui X, Jing X, Wu X, Yan M, Li Q, Shen Y, et al. DNA methylation in spermatogenesis and male infertility. Exp Ther Med 2016;12: 1973-9.

4. Camprubi C, Pladevall M, Grossmann M, Garrido N, Pons MC, Blanco J. Semen samples showing an increased rate of spermatozoa with imprinting errors have a negligible effect in the outcome of assisted reproduction techniques. Epigenetics 2012;7: $1115-24$.

5. Yanagisawa Y, Ito E, Yuasa Y, Maruyama K. The human DNA methyltransferases DNMT3A and DNMT3B have two types of promoters with different CpG contents. Biochim Biophys Acta 2002; 1577:457-65.

6. Messerschmidt DM, Knowles BB, Solter D. DNA methylation dynamics during epigenetic reprogramming in the germline and preimplantation embryos. Genes Dev 2014;28:812-28.

7. Kato Y, Nozaki M. Distinct DNA methylation dynamics of spermatogenic cell-specific intronless genes is associated with $\mathrm{CpG}$ content. PLoS One 2012;7:e43658.

8. Cortessis VK, Thomas DC, Levine AJ, Breton CV, Mack TM, Siegmund KD, et al. Environmental epigenetics: prospects for studying epigenetic mediation of exposure-response relationships. Hum Genet 2012;131:1565-89.

9. Owen CM, Segars JH Jr. Imprinting disorders and assisted reproductive technology. Semin Reprod Med 2009;27:417-28.

10. Li L, Le F, Wang LY, Xu XR, Lou HY, Zheng YM, et al. Normal epigenetic inheritance in mice conceived by in vitro fertilization and embryo transfer. J Zhejiang Univ Sci B 2011;12:796-804.

11. Daxinger $L$, Whitelaw $E$. Transgenerational epigenetic inheritance: more questions than answers. Genome Res 2010;20: 1623-8.

12. Latham KE, Schultz RM. Embryonic genome activation. Front Biosci 2001;6:D748-59.

13. Aoki VW, Emery BR, Carrell DT. Global sperm deoxyribonucleic acid methylation is unaffected in protamine-deficient infertile males. Fertil Steril 2006;86:1541-3.

14. Olszewska M, Barciszewska MZ, Fraczek M, Huleyuk N, Chernykh VB, Zastavna D, et al. Global methylation status of sperm DNA in carriers of chromosome structural aberrations. Asian J Androl 2017; 19:117-24.

15. Benchaib M, Braun V, Ressnikof D, Lornage J, Durand P, Niveleau $A$, et al. Influence of global sperm DNA methylation on IVF results. Hum Reprod 2005;20:768-73.

16. Menkveld R. Clinical significance of the low normal sperm morphology value as proposed in the fifth edition of the WHO Laboratory Manual for the Examination and Processing of Human Semen. Asian J Androl 2010;12:47-58. 
17. Talebi AR, Khalili MA, Hossaini A. Assessment of nuclear DNA integrity of epididymal spermatozoa following experimental chronic spinal cord injury in the rat. Int J Androl 2007;30:163-9.

18. Rosenborg L, Rao KM, Bjorndahl L, Kvist U, Pousette A, Akerlof E, et al. Changes in human sperm chromatin stability during preparation for in-vitro fertilization. Int J Androl 1990;13:287-96.

19. Talebi AR, Moein MR, Tabibnejad N, Ghasemzadeh J. Effect of varicocele on chromatin condensation and DNA integrity of ejaculated spermatozoa using cytochemical tests. Andrologia 2008;40:245-51.

20. Agarwal A, Bragais FM, Sabanegh E. Assessing sperm function. Urol Clin North Am 2008;35:157-71.

21. Rahiminia T, Hosseini A, Anvari M, Ghasemi-Esmailabad S, Talebi AR. Modern human sperm freezing: effect on DNA, chromatin and acrosome integrity. Taiwan J Obstet Gynecol 2017;56:472-6.

22. Talebi AR, Khalili MA, Vahidi S, Ghasemzadeh J, Tabibnejad N. Sperm chromatin condensation, DNA integrity, and apoptosis in men with spinal cord injury. J Spinal Cord Med 2013;36:140-6.

23. Talebi AR, Vahidi S, Aflatoonian A, Ghasemi N, Ghasemzadeh J, Firoozabadi RD, et al. Cytochemical evaluation of sperm chromatin and DNA integrity in couples with unexplained recurrent spontaneous abortions. Andrologia 2012;44 Suppl 1:462-70.

24. Nallella KP, Sharma RK, Aziz N, Agarwal A. Significance of sperm characteristics in the evaluation of male infertility. Fertil Steril 2006;85:629-34.

25. World Health Organization. WHO laboratory manual for the examination and processing of human semen. Geneva: World Health Organization; 2010.

26. Agarwal A, Said TM. Role of sperm chromatin abnormalities and DNA damage in male infertility. Hum Reprod Update 2003;9: 331-45.

27. Shamsi MB, Imam SN, Dada R. Sperm DNA integrity assays: diagnostic and prognostic challenges and implications in management of infertility. J Assist Reprod Genet 2011;28:1073-85.

28. Sakkas D, Moffatt O, Manicardi GC, Mariethoz E, Tarozzi N, Bizzaro D. Nature of DNA damage in ejaculated human spermatozoa and the possible involvement of apoptosis. Biol Reprod 2002;66:
1061-7.

29. Marques CJ, Joao Pinho M, Carvalho F, Bieche I, Barros A, Sousa M. DNA methylation imprinting marks and DNA methyltransferase expression in human spermatogenic cell stages. Epigenetics 2011;6:1354-61.

30. La Salle S, Trasler JM. Dynamic expression of DNMT3a and DNMT3b isoforms during male germ cell development in the mouse. Dev Biol 2006;296:71-82.

31. Cheng P, Chen H, Zhang RP, Liu SR, Zhou-Cun A. Polymorphism in DNMT1 may modify the susceptibility to oligospermia. Reprod Biomed Online 2014;28:644-9.

32. Pacheco SE, Houseman EA, Christensen BC, Marsit CJ, Kelsey KT, Sigman $\mathrm{M}$, et al. Integrative DNA methylation and gene expression analyses identify DNA packaging and epigenetic regulatory genes associated with low motility sperm. PLoS One 2011;6: e20280.

33. Yu B, Zhou H, Liu M, Zheng T, Jiang L, Zhao M, et al. Epigenetic alterations in density selected human spermatozoa for assisted reproduction. PLoS One 2015;10:e0145585.

34. Barzideh J, Scott RJ, Aitken RJ. Analysis of the global methylation status of human spermatozoa and its association with the tendency of these cells to enter apoptosis. Andrologia 2013;45:4249.

35. Aoki VW, Emery BR, Liu L, Carrell DT. Protamine levels vary between individual sperm cells of infertile human males and correlate with viability and DNA integrity. J Androl 2006;27:890-8.

36. Novakovic B, Wong NC, Sibson M, Ng HK, Morley R, Manuelpillai $U$, et al. DNA methylation-mediated down-regulation of DNA methyltransferase-1 (DNMT1) is coincident with, but not essential for, global hypomethylation in human placenta. J Biol Chem 2010;285:9583-93.

37. Kitamura A, Miyauchi N, Hamada H, Hiura H, Chiba H, Okae H, et al. Epigenetic alterations in sperm associated with male infertility. Congenit Anom (Kyoto) 2015;55:133-44.

38. Zama AM, Uzumcu M. Epigenetic effects of endocrine-disrupting chemicals on female reproduction: an ovarian perspective. Front Neuroendocrinol 2010;31:420-39. 